\title{
A VARIATIONAL PRINCIPLE IN KREIN SPACE
}

\author{
PAUL BINDING AND BRANKO NAJMAN
}

\begin{abstract}
A variational characterization, involving a max-inf of the Rayleigh quotient, is established for certain eigenvalues of a wide class of definitizable selfadjoint operators $Q$ in a Krein space. The operator $Q$ may have continuous spectrum and nonreal and nonsemisimple eigenvalues; in particular it may have embedded eigenvalues. Various applications are given to selfadjoint linear and quadratic eigenvalue problems with weak definiteness assumptions.
\end{abstract}

\section{INTRODUCTION}

Variational characterizations of eigenvalues have been studied for over a century and have been applied to comparison principles and numerical algorithms for a wide range of problems. For example, the generalised eigenvalue problem

$$
A x=\lambda B x
$$

with selfadjoint operators $A$ and $B$ in a Hilbert space $H$, can be studied in the "right definite case" (where $B$ is positive definite) via the equation

$$
B^{-1} A x=\lambda x \text {. }
$$

Formally, $B^{-1} A$ is selfadjoint in a "weighted" Hilbert space $H_{B}$ with inner product generated by

$$
(x, y)_{B}=(x, B y)
$$

and the variational principles that we consider involve the Rayleigh quotient

$$
r(x)=\frac{\left(x, B^{-1} A x\right)_{B}}{(x, x)_{B}}=\frac{(x, A x)}{(x, B x)}
$$

which can be evaluated directly from the operators $A$ and $B$. However, if $B$ is not definite then (1.4) vanishes for some nonzero $x$, so the usual variational principles need modification. If $A$ is definite (this is the so-called "left definite" problem) then (1.2) can be replaced by $A^{-1} B x=\lambda^{-1} x$ in a Hilbert space $H_{A}$.

Received by the editors March 25, 1991.

1991 Mathematics Subject Classification. Primary 49G05, 47B15, 35J20.

Key words and phrases. Minimax principle for eigenvalues, selfadjoint operators in Krein space, spectral functions, elliptic problems with indefinite weight.

Research of the first author supported by NSERC of Canada.

Research of the second author supported in part by the Ministry of Science of Croatia and was carried out while he was visiting the Department of Mathematics and Statistics, University of Calgary. 
See, e.g., [20] for a variational treatment of various boundary value problems by these techniques.

Problems which are neither left nor right definite have attracted considerable attention in recent years (cf. [9, 12] and their references), and it turns out that they have properties different from those of definite cases. The variational characterization of eigenvalues in such problems has however remained open despite early work of Richardson [16] for left definite, and a conjecture [17] for general, Sturm-Liouville problems, that the solution "for $m$ large enough, may be regarded as furnishing a minimum of a calculus of variations problem". Richardson does not explicitly state which problem, but it is clear from the context that he means minimization of $(x, A x)$ subject to $(x, B x)= \pm 1$ and $m$ (recursively defined) linear constraints of the form $\left(y_{i}^{ \pm}, B x\right)=0$. Nor is he clear about "large enough", but he cites his [17, §4] which shows that, for large enough $m$, there are precisely two eigenfunctions $y_{m}^{ \pm}$which oscillate $m$ times (one for each sign of $\lambda$-see [17, Theorem VII et prec.]). Our results show that each such eigenfunction obeys a double extremum principle, including the above recursive one.

If $B$ is $1-1$ then $H_{B}$, with the inner product generated by (1.3), becomes a Krein space. An early variational principle in this setting is due to Phillips [14]. It characterizes eigenvalues of a positive compact operator in $H_{B}$ by triple extrema involving sign-definite subspaces and thus it implicitly involves the positive and negative cones

$$
C^{ \pm}=\left\{x \in H_{B}: \pm(x, x)_{B}>0\right\} .
$$

This theory was extended to noncompact nonnegative operators by Textorius [18]. Various authors have also used these cones explicitly; for example, it was noted in [1] that (single) extrema of $r$ in (1.4) over $C^{ \pm}$give principal eigenvalues for certain left definite problems. Double extrema involving $C^{ \pm}$ have been used in [10 and 13] for some of the real eigenvalues $\lambda$ for indefinite matrix problems (1.1). Slightly different double (and also triple) extremal characterizations were established in [2] for elliptic equations with indefinite weights. Here we shall use spectral functions of definitizable selfadjoint operators in Krein spaces [11] to establish a double extremum principle for some of the eigenvalues under conditions which allow continuous spectrum, and in particular embedded eigenvalues corresponding to critical points (see §2). Our work includes all previous results that we know of.

In $\S 2$ we introduce our assumptions and state the main result, which is proved in $\S 3$. We conclude with $\S 4$ where applications to differential operators are discussed. In particular, we give a detailed comparison with [2] which uses two parameter ideas for statements and proofs, and we also show how some asymptotic estimates of [8] may be extended from the left definite case.

We conclude this section with some brief remarks on the application of Theorem 2.3 to problems of the form (1.1). Our assumptions are satisfied if, for example, $A$ is bounded below with compact resolvent and $B$ is injective and $A$-form compact, cf. [15]. Indeed, replacing $A$ by $A-\nu B$ for some real $\nu$ if necessary, we see that the space $H_{A}$ is a Pontryagin space and the verification of our hypotheses is straightforward.

The selfadjoint quadratic eigenvalue problem

$$
\left(\mu^{2} R+\mu S+T\right) y=0, \quad 0 \neq y \in X,
$$


can be transformed to (1.1) (see [9]) by defining $H=X \times X, \lambda=1 / \mu-\mu$,

$$
A=\left[\begin{array}{cc}
S & -R-T \\
-R-T & S
\end{array}\right] \text { and } B=\left[\begin{array}{cc}
-T & 0 \\
0 & R
\end{array}\right]
$$

on appropriate domains. If $S$ is bounded below with compact resolvent and $T, R$ are injective $S$-form compact operators then the above remarks lead to a max-inf characterization of eigenvalues of the problem (1.6). The above setting includes the well-known overdamped case from continuum mechanics which leads to a left-definite problem. We also cite Weinberger's Rayleigh quotient variational principle [19] for a quadratic problem with right-definite linearization.

\section{BACKGROUND AND BASIC RESULTS}

2.1 Cones. Throughout $\S \S 2$ and $3, Q$ is a selfadjoint operator in a Krein space $K$, with a (generally indefinite) inner product [, ]-cf. [3 and 7] for basic terminology etc.

Let $C$ be a cone in $K$, so $\lambda C \subseteq C$ for all $\lambda>0$. We define the internal dimension $d(C)$ to be the supremum of the dimensions of all subspaces in $C \cup\{0\}$. As in (1.5) we define the positive and negative cones of $K$ by

$$
C^{ \pm}=\{x \in K: \pm[x, x]>0\}, \quad \text { with } C_{0}^{ \pm}=C^{ \pm} \cup\{0\} .
$$

Note that $K$ is a Pontryagin space if at least one of $d\left(C^{ \pm}\right)$is finite. For any real number $\nu$ we also define the cone

$$
\Gamma_{\nu}^{-}=\left\{x \in D(Q):\left[x, Q_{\nu} x\right] \leq 0\right\}
$$

where $Q_{\nu}:=Q-\nu I$.

We shall make three assumptions on $Q$, the first being

(I) For some real $\nu,(\alpha) Q_{\nu}$ is (boundedly) invertible and $(\beta) d\left(\Gamma_{\nu}^{-}\right)$is finite.

Assumption $(\mathrm{I} \alpha)$ guarantees that $\sigma(Q)$ does not contain $\mathbb{R}$. If $\sigma(Q)$ contains $\mathbb{R}$, then the variational principle below is vacuous. Also, $(\mathrm{I} \beta)$ means that $[x, Q x]-\nu[x, x]$ has finitely many negative squares on $D(Q)$.

When $\sigma(Q)$ is discrete, our preparations are relatively simple and we shall discuss this case first. In particular, our remaining assumptions (and $(\mathrm{I} \alpha)$ ) are automatic.

Let $G_{\lambda}=N\left(Q_{\lambda}\right)$ be the geometric eigenspace at $\lambda$. If $G_{\lambda} \subset C_{0}^{+}$(resp. $C_{0}^{-}$), we say that $\lambda$ is of positive (resp. negative) type. Here $C_{0}^{ \pm}$come from (2.1). Developing this, we define the positive and negative multiplicities of an eigenvalue $\lambda$ by $d^{ \pm}(\lambda)=d\left(G_{\lambda} \cap C^{ \pm}\right)$. Writing $N^{+}$(resp. $N^{-}$) for the set of eigenvalues which are not of positive (resp. negative) type, we define

$$
\theta_{p}=\sup \left(N^{+} \cap \mathbb{R}\right) \text {. }
$$

Recall that, for any eigenvalue $\lambda$, the algebraic eigenspace $A_{\lambda}$ is the span of the Jordan chains $\left(y_{1}, \ldots, y_{l}\right)$ of length $l$, where $0 \neq y_{1} \in G_{\lambda}$ and

$$
Q_{\lambda} y_{j}=y_{j-1}, \quad 2 \leq j \leq l \text {. }
$$

It is easily seen that $\left[y_{1}, y_{1}\right]=0$ if $l>1$ or if $\lambda \notin \mathbb{R}$, so such $\lambda \in N^{+} \cap N^{-}$. Moreover it follows from (I $\beta)$ that the sum of the lengths $l$, over such $\lambda$, must be finite. 
Defining the subspace $F$ as the sum of the $A_{\lambda}$ for all $\lambda \in N^{-}$, and writing $\theta$ for $\theta_{p}$, which is now $\max \left(N^{+} \cap \mathbb{R}\right)$, we may then proceed directly to Proposition 2.2 below.

2.2 Spectral functions with critical points. We proceed now to discuss the case when $\sigma(Q)$ is not discrete. For the reader's convenience, we have assembled some basic definitions and properties related to definitizable selfadjoint operators on Krein spaces. For further details see [3, $\S 4.1],[7, \S$ VIII.6] and especially [11]. A selfadjoint operator $Q$ is definitizable if its spectrum $\sigma(Q)$ is not the whole complex plane, and if a nonconstant definitizing polynomial $\pi$ exists so that $\pi(Q)$ is nonnegative definite. Such an operator has a spectral function $E$, i.e., an orthoprojector-valued function defined on real intervals whose endpoints are not zeros of $\pi$, together with the complements of such intervals in $\mathbb{R}$. Then $\pm E(\Delta)$ is positive definite if $\pm \pi$ is positive on $\Delta \cap \sigma(Q)$, and

$$
Q x=\int_{\Delta} \lambda d E(\lambda) x \text { for all } x \in E(\Delta) K .
$$

Here and below, $\Delta$ will always denote a set on which $E$ is defined as above. $E$ may be extended to further subsets of $\mathbb{R}$, but the above definition suffices for our purposes. We note that

$$
\sigma\left(\left.Q\right|_{E(\Delta) K}\right) \subset \bar{\Delta}
$$

so $\left.Q\right|_{E(\Delta) K}$ is invertible if $0 \notin \bar{\Delta}$.

Proposition 2.1. $Q$ is definitizable, and hence admits a spectral function $E$.

Proof. In the case $\nu=0$, this is a result of Langer [11], who constructs an "equivalent" selfadjoint operator on an auxiliary Pontryagin space. In our case we use $Q_{\nu}$ instead of $Q$, and we obtain a definitizing polynomial of the form

$$
\pi(\lambda)=(\lambda-\nu)|p(\lambda)|^{2}
$$

for some polynomial $p$.

We term $\lambda \in \mathbb{R} \cup\{\infty\}$ a critical point of $Q$, and we write $\lambda \in c(Q)$, if $E(\Delta)$ is indefinite whenever $\lambda \in$ int $\Delta$. Necessarily then $\lambda \in \sigma(Q)$. Also $\infty \neq \lambda \in c(Q)$ implies $\pi(\lambda)=0$ by the above, so there are only finitely many critical points. A critical point is regular if there are $\alpha>0$ and a neighbourhood $\Delta_{0}$ of $\lambda$ so that $\|E(\Delta)\| \leq \alpha$ whenever $\lambda \in \Delta \subset \Delta_{0}$. This is the case, for example, if $\lambda$ is an isolated point of $\sigma(Q)$.

We define $\lambda \in \sigma(Q)$ to be of positive (resp. negative) type if $E(\Delta) K \subset C_{0}^{+}$ (resp. $C_{0}^{-}$) for some $\Delta$ containing $\lambda$. This extends our previous definition to nonisolated $\lambda$. As before, $\lambda \in N^{+}$(resp. $N^{-}$) if $\lambda$ is not of positive (resp. negative) type.

Note that $\nu$ is not a critical point of $Q$ by $(\mathrm{I} \alpha)$, but that $\infty$ is a critical point in general. Using $(2.5)$ we see that $\lambda>\nu$ (resp. $\langle\nu)$ is a finite critical point iff $p(\lambda)=0$ and $\lambda$ is an eigenvalue not of definite type, i.e., $\lambda \in N^{+} \cap N^{-}$.

Our next assumption is

(II) The finite critical points of $Q$ are all regular.

Regularity is automatic for isolated eigenvalues. Suppose now that all eigenvalues of positive type exceed $\nu$. In this case we define $\theta$ as the greater of $\theta_{c}$ and $\theta_{p}$, where $\theta_{c}$ is the supremum of the continuous spectrum $<\nu$, and $\theta_{p}$ 
comes from (2.2). It follows from (II) that $\sum A_{\lambda}$ is nondegenerate (the sum being over $\lambda \in N^{-}$) but this is in general infinite dimensional. The following refinement uses an orthogonal decomposition $A_{\lambda}=F_{\lambda}[\dot{+}] H_{\lambda}$ given in [6], where $F_{\lambda}$ is finite dimensional and nondegenerate, and where $H_{\lambda} \subseteq G_{\lambda}$ is a Hilbert space under [ , ]. Specifically, defining $F$ as the sum of the $A_{\lambda}$ for nonreal $\lambda$ and $F_{\lambda}$ for real $\lambda \in N^{-}$, we may proceed to Proposition 2.2.

Finally, suppose $\theta>\nu$, so $\theta$ is simply the maximum (by $(\mathrm{I} \beta)$ ) of the eigenvalues $\lambda>\nu$ with $\lambda \in N^{+}$. In this case we need a third assumption

(III) $\sigma(Q) \cap[\nu, \theta]$ consists of finitely many eigenvalues of finite multiplicity.

We remark, by virtue of $(\mathrm{I} \beta)$ and our previous comments, that multiplicity here could be interpreted as "positive", "geometric" or "algebraic". To the previous definition of $F$ we then add the sum of the $A_{\lambda}$ over $\lambda \in[\nu, \theta]$.

Proposition 2.2. $F$ defined as above, is finite dimensional, nondegenerate and invariant for $Q$. Moreover all real eigenvalues of $\left.Q\right|_{F}$ are $\leq \theta$, while $\left.Q\right|_{F \perp}$ has real spectrum, of negative type $<\theta$ and of positive type $>\theta$.

Proof. The only nontrivial contention is nondegeneracy of $F$, which is covered by the arguments of $[6, \S 5]$.

We remark that the results of Propositions 2.1 and 2.2 could be used instead of (I)-(III).

2.3 A variational principle. We list those eigenvalues $\lambda>\theta$, for which $d^{+}(\lambda)>0$, in nondecreasing order and counted by positive multiplicity, as $\lambda_{1}^{+} \leq \lambda_{2}^{+} \leq \cdots$. Our main result states that these eigenvalues may be characterized by a max-inf principle with shifted index. More precisely, recalling Proposition 2.2 and defining $d^{ \pm}=d\left(F \cap C^{ \pm}\right)$and

$$
\mu_{j}=\max \left\{\inf \left\{[x, Q x] /[x, x]: x \in M \cap C^{+} \cap D(Q)\right\}: M \in \mathscr{M}_{j}\right\}
$$

where $\mathscr{M}_{j}$ is the set of subspaces of $K$ of codimension $j-1$, we have

Theorem 2.3. Assume (I)-(III). Then $\lambda_{j}^{+}=\mu_{j+d^{+}}, j=1,2, \ldots$.

Remark 2.4. If $C^{+}$is replaced by $C^{-}$in (2.6) then corresponding eigenvalues $\lambda_{j}^{-}$with eigenvectors in $C^{-}$can be characterized. Similarly, if the definition of $\theta$ and (II) are modified in an obvious way then $\lambda_{j}^{ \pm}$may be listed in nonincreasing order and all those below a certain $\theta^{\prime}$ can be characterized.

Remark 2.5. When $\operatorname{dim} K$ is finite, Theorem 2.2 is equivalent to a result in [13]. In fact the setting of (1.1), where $K=H_{B}$ and $Q=A^{-1} B$ with $B$ invertible, is equivalent to the one here. Note that $[x, Q x] /[x, x]=r(x)$ of (1.4) while (I)-(III) are automatic in finite dimensions. Also the other formulae for $\lambda_{j}^{ \pm}$mentioned in Remark 2.4 can be read from [13] (with dimensions there replaced by appropriate codimensions).

Similarly, in the left-definite case of (1.1), $Q$ is a uniformly positive operator in $K$ and so (I)-(III) are automatic with $\theta=\nu=0$. In the right-definite case we recover the ordinary Weyl-Courant principle in a Hilbert space.

Remark 2.6. For any Jordan chain $\left(y_{1}, \ldots, y_{l}\right)$ (see (2.3)), an easy calculation shows that $\delta=\left[y_{1}, y_{l}\right]$ is real. If $\delta<0$ then we say that the chain has negative sign ( $\operatorname{sgn} \delta$ is associated with various terms in the literature, e.g. inertial signature and sign characteristic). It turns out that negative sign Jordan chains 
of length $l=2$ may be ignored in the calculation of $\theta$. This improves Theorem 2.3 and follows from arguments in [13] and $\S 3$.

Remark 2.7. If the $\lambda_{j}^{+}$cease to exist from some $j$ on, the remaining $\mu_{j+d^{+}}$ characterize the infimum of the continuous spectrum $>\theta$.

\section{PROOF OF THE VARIATIONAL PRINCIPLE}

For notational ease we fix $j$ and write $\lambda=\lambda_{j}^{+}$.

Lemma 3.1. There are $d^{ \pm}$-dimensional subspaces $F^{ \pm}$of $F$ such that $F^{+} \subset C_{0}^{+}$ and

$$
\pm\left[x, Q_{\lambda} x\right] \leq 0 \text { for all } x \in F^{ \pm} .
$$

Proof. Since $\lambda>\theta$, Proposition 2.2 shows that $\left.Q\right|_{F}$ has no (real) eigenvalues $>\lambda$. The result now follows from [13, Steps 1 and 2], which applies since $F$ is nondegenerate.

We shall base the proof on the following elementary decomposition principle.

Lemma 3.2. If (i) $[y, z]=0=[y, Q z]$ for all $y \in Y, z \in Z$ where $Y, Z \subseteq$

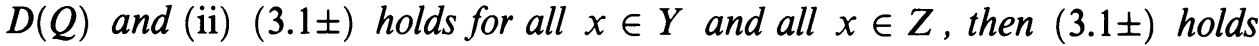
for all $x \in Y+Z$.

Now let $x_{l} \in C^{+}$satisfy $\left[x_{l}, x_{l}\right]=1$ and form a linearly independent set of eigenvectors for $\lambda_{l}^{+}, l=1, \ldots, j$, and write $S_{j}$ for the $\operatorname{span} \operatorname{sp}\left\{x_{1}, \ldots, x_{j}\right\}$.

Lemma 3.3. $\theta_{d^{+}+j} \leq \lambda$.

Proof. Since $S:=F^{+}+S_{j}$ is a $\left(d_{+}+j\right)$-dimensional subspace of $C_{0}^{+}$, for any $M \in \mathscr{M}_{d^{+}+j}$ there is $x \in M \cap S \cap C^{+}$. Evidently (3.1+) holds on $S_{j}$, so by Lemmas 3.1 and 3.2, $(3.1+)$ holds on $S$ and $\mu_{d^{+}+j} \leq[x, Q x] /[x, x] \leq \lambda$.

Since $S_{j-1}=\operatorname{sp}\left\{x_{1}, \ldots, x_{j-1}\right\} \subset C_{0}^{+}, F+S_{j-1}$ is nondegenerate, and hence has an orthocomplement $T$ in $K$ [7, Corollary I.11.9]. Let $E_{T}$ be the spectral function of $\left.Q\right|_{T}$. Fix $\omega>0$ so that $\left.\Omega:=\right]-\omega, \omega$ [ contains $\lambda, \nu$ and the zeros of $p$ (2.5).

Lemma 3.4. (3.1-) holds for all $x \in E_{T}(\Omega) K$.

Proof. Since $\lambda>\theta$, Proposition 2.2 shows that $\left[x, E_{T}(\cdot) x\right]$ is nondecreasing on $] \lambda, \infty[$. Similarly the construction of $F$ and the definition of $T$ show that $\left[x, E_{T}(\cdot) x\right]$ is nonincreasing on $]-\infty, \lambda[$. Thus

$$
\int_{-\omega}^{\omega} \mu[x, E(d \mu) x] \geq \lambda \int_{-\omega}^{\omega}[x, E(d \mu) x],
$$

whence $[x, Q x] \geq \lambda[x, x]$, for any $x \in E_{T}(\Omega) K$.

Lemma 3.5. (3.1-) holds for all $x \in P K$ where $P=E(\overline{\mathbb{R}} \backslash \Omega)$.

Proof. For all real $t \notin \Omega, \varphi(t):=(t-\lambda) /(t-\nu)>0$. By $(2.5),\left[x, Q_{\nu} x\right] \geq 0$ for all $x \in R(p(Q))$ and so

$$
\left[Q_{\lambda} x, x\right]=\left[\varphi(Q) Q_{\nu} x, x\right] \geq 0
$$

whenever $x=p(Q) y$ with $y \in D(p(Q) P)$. Noting that $p(\lambda)$ is bounded away from zero for all $\lambda \notin \Omega$, we see that $\left.p(Q) P\right|_{P K}$ is invertible, cf. (2.4), so (3.2) holds for all $x \in P K$. 
We are now in a position to complete the proof of Theorem 2.3. By Lemmas 3.2, 3.4, and 3.5, $(3.1-)$ holds on $E_{T}(\overline{\mathbb{R}}) K=T$. By Lemmas 3.1 and 3.2, then, (3.1-) holds on

$$
M_{j}=F^{-}+T
$$

which belongs to $\mathscr{M}_{d^{+}+j}$ since $F$ is nondegenerate. Thus

$$
\mu_{d^{+}+j} \geq \inf \left\{[x, Q x] /[x, x]: x \in M_{j} \cap C^{+}\right\} \geq \lambda,
$$

which, with Lemma 3.3, establishes our theorem.

Remark 3.6. If $N$ is a subspace of $K$ such that $M_{j} \cap N=\{0\}$, where $M_{j}$ is the maximising subspace of (3.3), then Theorem 2.3 gives

$\lambda_{j}^{+}=\max \left\{\inf \left\{[x, Q x] /[x, x]: x \in M \cap D(Q) \cap C^{+}\right\}: M \in \mathscr{M}_{j+d^{+}}, M \cap N=\{0\}\right\}$.

Since [13] one can ensure that $F^{+} \cap F^{-}=\{0\}$, we can choose

(i) $\operatorname{dim} N=d^{+}-\sum_{\lambda \leq \theta} d^{+}(\lambda)$, and

(ii) $N \subseteq F$,

so $N$ is spanned by root vectors of $\left.Q\right|_{F}$.

This will be used in 4.3. Note that (i) cannot be relaxed to $\operatorname{dim} N>d^{+}$.

\section{ELLIPTIC EQUATIONS WITH INDEFINITE WEIGHTS}

4.1 Setting. Let $\Omega$ be a bounded smooth domain in $\mathbb{R}^{n}$ and $q, w \in L^{\infty}(\Omega)$. We consider the eigenvalue problem

$$
(-\Delta+q) y=\lambda w y, \quad y=0 \quad \text { on } \partial \Omega .
$$

As in [2], a (nonzero) function $y \in H:=W_{0}^{1,2}(\Omega)$ satisfying (4.1) is an eigenfunction for the eigenvalue $\lambda \in \mathbb{C}$; moreover, $-\Delta+q$ can be replaced by a uniformly elliptic operator and the Dirichlet condition by a more general selfadjoint boundary condition. Actually our Krein space setting is sufficiently general to handle continuous spectrum (thus allowing unbounded $\Omega$ and/or singular coefficients and weight function $w$ ). We adhere to the problem (4.1) and the above restrictive assumptions on $Q$ and $w$ for simplicity and also to facilitate a detailed comparison with [2]. We impose the assumption

$$
\{x: w(x)=0\} \text { is of Lebesgue measure zero }
$$

which is more stringent than the corresponding assumption of [2]; on the other hand we do not need the unique continuation assumption of [2]. The only reason we impose (4.2) is to form the Krein space $K$ below, and moreover we hope to show elsewhere how to remove (4.2) entirely.

Let $L$ be the selfadjoint realization of $-\Delta+q$, with Dirichlet boundary condition, in $L^{2}(\Omega)$, so $D(L)=W^{2,2}(\Omega) \cap H$. From [5, Lemma 2.1] we can translate the $\lambda$-origin if necessary to ensure that $L$ has a compact inverse. Note also that $L$ is semibounded, and the bilinear form associated with $L$ is given by

$$
l(y, z)=\int_{\Omega}(\nabla y \cdot \nabla \bar{z}+q y \bar{z}) \quad \text { on } D(l)=H .
$$


4.2 Application of Theorem 2.3. Let $K$ be the space $L_{|w|}^{2}$ (consisting of functions $y$ for which $\|y\|_{|w|}^{2}=\int_{\Omega}|w||y|^{2}$ is finite) endowed with the (possibly indefinite) inner product

$$
[y, z]=\int_{\Omega} w y \bar{z} .
$$

Then $K$ is a Krein space. Since $w \in L^{\infty}(\Omega)$, it is clear that $L^{2}(\Omega)$ is continuously embedded in $K$. Let

$$
D(Q)=\left\{y \in D(L):|w|^{-1 / 2} L y \in L^{2}(\Omega)\right\}, Q y=w^{-1} L y \quad \text { if } y \in D(Q) .
$$

Clearly $Q$ is an operator in $K$. In fact we have

Theorem 4.1. The operator $Q$ is a semibounded selfadjoint operator in $K$ with compact inverse.

Proof. First if $x_{j} \rightarrow 0$ in $K$ then $Q^{-1} x_{j}=L^{-1} w x_{j} \rightarrow 0$ in $L^{2}(\Omega)$, hence in $K$. Thus $Q$ is boundedly invertible. Next we show that

$$
Q \text { is densely defined in } K \text {. }
$$

Assume $x$ is orthogonal to $D(Q)$ in $K$. Then

$$
\left[x, Q^{-1} y\right]=\int_{\Omega} w L^{-1}(w y) \bar{x}=0 \text { for all } y \in K .
$$

Since $(\operatorname{sgn} w)|w|^{1 / 2}$ is an isomorphism of $K$ onto $L^{2}(\Omega)$, the selfadjointness of $L$ shows that $|w|^{1 / 2} L^{-1}(w x)=0$ a.e. in $\Omega$, whence $x=0$.

For any $y, z \in D(Q)$ we have $[Q y, z]=\int_{\Omega}(L y) \bar{z}$ so $Q$ is symmetric and semibounded since $D(Q) \subset D(L)$. Let $z \in D\left(Q^{*}\right)$. Then the functional $\varphi: y \mapsto[Q y, z]=\int_{\Omega}(L y) \bar{z}$ defined on $D(Q)$ is continuous in $K$ and therefore in $L^{2}(\Omega)$. An argument similar to the one for (4.3) shows that $D(Q)$ is dense in $H=W_{0}^{1,2}(\Omega)$ and hence also in $L^{2}(\Omega)$. As $L$ is selfadjoint we conclude that $z \in D(L)$. Therefore $\varphi: y \mapsto \int_{\Omega} y(\overline{L z})$ is continuous in $L_{|w|}^{2}$, so $z \in D(Q)$, and $Q$ is selfadjoint in $K$.

Finally let $x_{j}$ converge weakly to zero in $K$. Then $w x_{j}$ converge weakly to zero in $L^{2}(\Omega)$ so

$$
\left\|Q^{-1} x_{j}\right\|_{L_{|w|}^{2}}=\left\||w|^{1 / 2} L^{-1}\left(w x_{j}\right)\right\|_{L^{2}} \rightarrow 0
$$

which proves that $Q^{-1}$ is compact.

A Jordan chain for $(4.1)$ is a sequence $y_{j} \in H, j=0,1, \ldots$, such that $y_{0}=0$,

$$
(L-\lambda w) y_{j}=w y_{j-1}, \quad j=1, \ldots,
$$

with $y_{1}, \ldots$ linearly independent. We note the following easy result:

Lemma 4.2. The Jordan chains for (4.1) coincide with those for $Q$.

Proof. If $y_{1} \in D(Q)$ with $Q y_{1}=L y_{1}$ then $L y_{1}=\lambda w y_{1}$ since $D(Q) \subset D(L)$. Conversely, if (4.1) holds then $w^{-1} L y_{1}=\lambda y_{1} \in L^{2}(\Omega)$, so $|w|^{-1 / 2} L y_{1}$ $\in L^{2}(\Omega)$, hence $y_{1} \in D(Q)$ and $Q y_{1}=\lambda y_{1}$. The argument for $j>1$ is similar. 
By Theorem 4.1 and our earlier comments, (I)-(III) hold. Lemma 4.2 we may label the eigenvalues $>\theta$ for (4.1) according to positive multiplicity as $\lambda_{1}^{+} \leq \lambda_{2}^{+} \leq \cdots$. Since

$$
r(y)=\frac{[y, Q y]}{[y, y]}=\frac{\int_{\Omega}\left(|\nabla y|^{2}+q|y|^{2}\right)}{\int_{\Omega} w|y|^{2}}, \quad y \in D(Q),
$$

and since $D(Q)$ is dense in $H=D(l)$, Theorem 2.3 yields the following:

Corollary 4.3. There exists $\theta \in \mathbb{R}$ so that all eigenpairs $(\lambda, y)$ of (4.1) satisfying $\lambda=\lambda_{j}^{+}>\theta, y \in H$, also satisfy $\int_{\Omega} w|y|^{2}>0$, and moreover

$$
\lambda_{j}^{+}=\max \left\{\inf \left\{r(z): \int_{\Omega} w|z|^{2}>0, z \in M \cap H\right\}: M \in \mathscr{M}_{j+d^{+}}\right\} .
$$

4.3 Comparison with [2]. Allegretto and Mingarelli consider a two parameter embedding of (4.1), viz.

$$
(\Delta+\lambda w+\mu) y=0
$$

and they characterize certain eigenvalues labelled $\lambda_{i, k}$, where $\lambda_{i, k}=\lambda_{j}^{+}$under certain conditions. One condition is that $\lambda_{i, k}$ lies on the $k$ th $(\lambda, \mu)$ eigencurve of (4.4), i.e., that $\Delta+\lambda w$ has $k$ nonnegative eigenvalues, counted by multiplicity.

Analysis of the eigencurves (cf. [4]) shows that $k=j+d^{+}$whenever $\lambda_{i, k}>\theta$ so we may replace $(4.4)$ by

$$
\lambda_{i, k}=\max \left\{\inf \left\{r(z): \int_{\Omega} w|z|^{2}>0, z \in M \cap H\right\}: M \in \mathscr{M}_{k}\right\}
$$

in Corollary 4.3. This not only shows that the index shift in (4.4) is very natural, but also gives an alternative method for its calculation, involving eigenvalues $\mu$ of (4.5) for fixed $\lambda$, but not requiring knowledge of $F$.

[2, Corollary 2.10] contains a formula like (4.6) but with $M \in \mathscr{M}_{k}$ further constrained by $M \cap N=\{0\}$ where $N$ is a subspace of root vectors such that (i) $\operatorname{dim} N=k-i$, so $\operatorname{dim} N=d^{+}-(i-j)$, and (ii) $N$ is neutral, so $N \subseteq F$. Thus [2, Corollary 2.10] follows from (4.6) and Remark 3.6, provided $N$ there can be chosen neutral. This is possible if and only if all Jordan chains of $Q$ of odd length $>1$ have negative sign (see Remark 2.6). Moreover if there are no such Jordan chains then $N$ may also be used for similar characterizations of $\lambda_{j}^{-}$; cf. Remark 2.4 .

We observe in conclusion that $\lambda_{i, k}=\lambda_{j}^{+}$also depends on counting $i$ via positive multiplicities $d^{+}(\lambda)$, and one must interpret the remarks following [2, Theorem 2.8] in this light.

4.4 Asymptotics. Recently there has been significant interest in eigenvalue asymptotics and "counting functions" for left-definite problems. As an example we cite [8, Theorem 3.1], where the limit

$$
\lambda_{j}^{+} j^{-2 / n}\left\|w_{+}\right\|_{L^{n / 2}(\Omega)} \rightarrow(2 \pi)^{2}\left(V_{n}\right)^{-2 / n} \quad \text { as } j \rightarrow \infty
$$

is established, with $V_{n}$ as the volume of the unit ball in $\mathbb{R}^{n}$ and $w_{+}=\max \{0, w\}$. The key tools for this and similar estimates are

(a) corresponding formulae for right definite problems and 
(b) variational formulae for eigenvalues of left definite problems. Since (a) is independent of left definiteness and (b) is directly generalised by Corollary 4.3, we conclude

Corollary 4.4. (4.7) carries over to (4.1).

More general problems, as mentioned in 4.1, may also be treated following the remarks in [8].

\section{REFERENCES}

1. W. Allegretto and A. B. Mingarelli, On the nonexistence of positive solutions for a Schrödinger equation with an indefinite weight-function, C.R. Math. Rep. Acad. Sci. Canada 8 (1986), 69-73.

2. __ Boundary problems of the second order with an indefinite weight-function, J. Reine Angew. Math. 398 (1989), 1-24.

3. T. Ja. Azizov and I. S. Iohvidov, Linear operators in spaces with an indefinite metric, Wiley, 1989.

4. P. A. Binding and P. J. Browne, Applications of two parameter spectral theory to symmetric generalised eigenvalue problems, Applicable Anal. 29 (1988), 107-142.

5. P. A. Binding and K. Seddighi, On root vectors of self-adjoint pencils, J. Functional Anal. 70 (1987), 117-125.

6. P. A. Binding and B. Najman, Regularity of finite type critical points for self-adjoint operators in Krein space, preprint.

7. J. Bognár, Indefinite inner product spaces, Springer-Verlag, 1974.

8. J. Fleckinger and M. Lapidus, Eigenvalues of elliptic boundary value problems with an indefinite weight function, Trans. Amer. Math. Soc. 295 (1986), 305-324.

9. W. M. Greenlee, A quadratic eigenvalue problem, Proc. Amer. Math. Soc. 40 (1973), 123127.

10. P. Lancaster and Q. Ye, Variational properties and Rayleigh quotient algorithms symmetric matrix pencils, Vol. OT40, Birkhäuser-Verlag, 1989, pp. 247-278.

11. H. Langer, Spectral functions of definitizable operators in Krein spaces, Lecture Notes in Math., vol. 948, Springer-Verlag, 1982, pp. 1-46.

12. A. B. Mingarelli, A survey of the regular weighted Sturm-Liouville problem: the non-definite case, Proc. Workshop Appl. Differential Equations, World Scientific Publ., 1986, pp. 109137.

13. B. Najman and Q. Ye, A minimax characterization for eigenvalues of Hermitian pencils, Linear Algebra Appl. 144 (1991), 217-230.

14. R. S. Phillips, A minimax characterization for the eigenvalues of a positive symmetric operator in a space with an indefinite metric, J. Fac. Sci. Univ. Tokyo Sect. IA Math. 17 (1970), 51-59.

15. M. Reed and B. Simon, Methods of modern mathematical physics, vol. IV, Analysis of Operators, Academic Press, 1978.

16. R. G. D. Richardson, Das Jacobische Kriterium der Variationsrechnung und die Oszillationseigenschaften Linearer Differentialgleichungen 2 Ordnung, Math. Ann. 68 (1910), 279-304.

17. Contributions to the study of oscillation properties of the solutions of linear differential equations of the second order, Amer. J. Math. 40 (1918), 283-316.

18. B. Textorius, Minimaxprinzipe zur Bestimmung der Eigenwerte J-nichtnegativer Operatoren, Math. Scand. 35 (1974), 105-114. 
19. H. F. Weinberger, On a nonlinear eigenvalue problem, J. Math. Anal. Appl. 21 (1968), 506-509.

20. _ Variational methods for eigenvalue approximation, CBMS Regional Conf. Ser. in Math., vol. 15, SIAM, Philadelphia, Pa., 1974.

Department of Mathematics and Statistics, University of Calgary, 2500 University Drive N.W., Calgary, Alberta, Canada T2N 1N4

E-mail address: binding@acs.ucalgary.ca

Department of Mathematics, University of Zagreb, BijeničKa 30, 41000 Zagreb, CroATIA

E-mail address: najman@cromath.math.hr 